\title{
Biodegradability Features of Fluoxetine as a Reference Compound for Monitoring the Activity of Activated Sludges in Drug Biodegradation Studies
}

\author{
Yolanda Martín-Biosca ${ }^{1}$, Laura Escuder-Gilabert ${ }^{1 *}$, Mireia Perez-Baeza ${ }^{1}$, Salvador Sagrado $^{1,2}$, \\ María José Medina-Hernández ${ }^{*}$ \\ ${ }^{1}$ Departamento de Química Analítica, Universitat de València, Burjassot, Valencia, Spain \\ ${ }^{2}$ Instituto Interuniversitario de Investigación de Reconocimiento Molecular y Desarrollo Tecnológico (IDM). \\ Universitat Politècnica de València, Universitat de València. Burjassot, Valencia, Spain.
}

*Corresponding Author: Laura Escuder-Gilabert, Departamento de Química Analítica, Universitat de València, Burjassot, Valencia, Spain

\begin{abstract}
Drugs in the environment and its potential toxic effects are recognised problems in the environmental chemistry area. Drugs can be biodegraded in the wastewater treatment plants (WWTP) as a result of microbial processes. However, the degradation capability of the microbial community of an activated sludge could change over time. In OECD ready biodegradability tests (RBTs), the use of a reference compound that is usually easily and fast degraded is recommended in order to ensure that the microbial community in the test system is active. However, the biodegradability of most drugs is poor and slow compared with the classical proposed reference compounds. In this work, fluoxetine (among other drug candidates tested) was suggested as a consistent reference compound to control the activity of activated sludges in drug biodegradation studies. To characterize its biodegradability features, fluoxetine was assayed in OECD RBTs-recommended conditions. Experiments were performed using activated sludges taken at different dates from a local WWTP, different storage times, and by three different operators (intermediate precision study). A fluoxetine-biodegradation acceptance criterionthat could be connected to data from other drugs or comparison studies (different procedures, conditions, activated sludges) was proposed.
\end{abstract}

Keywords: Drugs Biodegradation, Biodegradability test, Fluoxetine, Reference compound, Intermediate precision study

\section{INTRODUCTION}

Drugs in the environment (mainly waterways) and its potential adverse effects are recognised problems in the environmental chemistry area [1,2]. Drug residues are assumed pseudopersistents in water environments due to their continual introduction into surface waters from wastewater treatment plants (WWTP)[1], where they can be biodegraded because of microbial processes.

Biodegradability assays are designed to test a chemical substance as the sole carbon source in a mixed inoculum [3]. The OECD ready biodegradability tests (RBTs) are used as screening methods to provide information about of the potential persistence of chemicals under most environmental conditions [4]. This risk parameter depends on the chemical structure of the compound as well as on the conditions under which the degradation process takes place. Drugs biodegradability studies are currently a priority in environmental studies [5-10]. This is due to their extensive use with a continuous release into the environment and the subsequent impact.

RBTs use natural populations of microorganisms to ensure the presence of a diversity of species able to degrade a wide variety of substrates. Activated sludges are a common inoculums source in RBTs due to their availability and high biodegradation potential [11]. Ideally, comparable results should be obtained using activated sludges from different wastewater treatment plants (WWTP). However, the microbial community of an activated sludge in a WWTP are sensitive to the ambient environment, the complexity of the biological and chemical reactions, and the large variations in the influent flow $[12,13]$. Therefore, activated sludge potential activity to degrade incoming compounds could be different at different times. Consequently, data of biodegradability obtained using a given inoculum reflect just the potential capability of this activated sludge to degrade pollutants in a specific moment under specific conditions. 
The use of surrogate microbial communities as certified biological materials with the same metabolic potential as an activated sludge has been proposed for several authors for quality control and to provide traceability [11]. However, biodegradation experiments inoculated with fresh activated sludge simulate in a more realistic way the complexity of microbial processes occurring in aquatic environments. As an alternative, the OECD recommend the use of a reference compound, which is usually easily degraded under the tested conditions, in order to ensure the activity of the microbial community in the test system [4].

For this purpose, aniline, sodium acetate and sodium benzoate have been recommended as reference compounds. Assays with the reference compound and the test compound have to be performed in parallel. However, these reference compounds all degrade in these methods even when no inoculum is deliberately added [4]. This becomes an inconvenient in practice since the total degradation could not be exclusively a biodegradation process (i.e. the real activity of the inoculum is not reflected). Moreover, the compounds recommended are simpler from a structural point of view and faster biodegraded than most of the target compounds (e.g. drugs). Moreover, the rapid biodegradation can make it difficult to obtain biodegradation curves for the reference compound, which could be useful in comparison studies (e.g. half-life estimations). Therefore, the recommended reference compounds could not be consistent for studies on drug biodegradation.

In this work, biodegradability studies of several drugs in OCDE RBTs-resembling conditions were performed, with the aim of selecting a suitable drug candidate (according to two predefined criteria) as potential reference compound. To the best of our knowledge, the use of such fit-for-purpose biodegradation reference drug has not been reported. Once this drug was selected, it was evaluated as a consistent control of the inoculum activity for future drug-biodegradability studies. For this purpose, a long-term characterization (intermediate precision study) and a short-term characterization (biodegradation profile) were performed. For the intermediate precision study, habitual sources of variation that could influence the results were used in biodegradation experiments. They include inoculums from activated sludges from a local WWTP taken at different dates, different laboratory length of inoculum storage before use and three operators to carry out the biodegradation and analytical procedures. To the best of our knowledge, a similar biodegradation study has not been reported in the literature.

\section{MATERIALS AND METHODS}

\subsection{Chemicals and Solutions}

All reagents were of analytical grade. Fluoxetine hydrochloride (FLX) was kindly donated by Alter (Madrid, Spain). Fenfluramine hydrochloride was kindly donated by Vrije Universiteit Brussel (Brussels, Belgium). Benzoic acid, aniline, labetalol hydrochloride, clemastine fumarate, nomifensine maleate, orphenadrine hydrochloride, terfenadine, trimeprazine hemi(+)-tartrate, methotrimeprazine maleate, promethazine hydrochloride and verapamil hydrochloride were from Sigma-Aldrich (@Merck KGaA, Darmstadt, Alemania). Metoprolol was from Alfa Aesar (Thermo Fisher Scientific Inc., Karlsruhe, Alemania) and propranolol was from Acros (Acros Organics, Geel, Bélgica).Stock standard solutions of $1000 \mathrm{mg} \cdot \mathrm{L}^{-1}$ were prepared by dissolving the adequate amount of compounds in methanol (®Multisolvent, HPLC grade; Scharlau, Barcelona, Spain).

For mobile phase preparation, Hydrochloric acid (37 \%), sodium dihydrogen phosphate monohydrate, sodium hydroxide and acetonitrile (®Multisolvent, HPLC grade; Scharlau) were used. For the biodegradability assays, working solutions of approximately $20 \mathrm{mg} \mathrm{L}^{-1}$ of compounds were prepared by dilution of the stock solution in minimal salts medium (MSM; described in [14]). Activated sludge samples came from the aerated tanks of a municipal WWTP (Quart Benàger, Valencia, Spain), whose technical details are reported elsewhere[14]. They were collected at different times in plastic flasks.Ultra Clear TWF UV deionized water (SG Water, Barsbüttel, Germany) was used to prepare solutions. All the solution were stored at $4{ }^{\circ} \mathrm{C}$ until usage.

\subsection{Biodegradability Assays}

Mixtures of approximately $20 \mathrm{mg} \cdot \mathrm{L}^{-1}$ of each compound inoculated with the activated sludge were prepared in $15 \mathrm{~mL}$-reactors (test tubes) as described elsewhere[14], including details on incubation, optical density measurements at $600 \mathrm{~nm}\left(O D_{600}\right)$, centrifugation and filtration. For the selection of the 
reference compound candidate, incubation samples were taken at 0 and 7 days. Abiotic degradation (in absence of inoculum) were also evaluated in parallel. All assays were performed by duplicate and the mean values were used except when it is indicated.

\subsection{HPLC Methods}

The separation of the compounds was performed using a $\mathrm{C}_{18}$ column (Halo; $2.7 \mu \mathrm{m}, 75 \times 4.6 \mathrm{~mm}$ i.d.; Advanced Materials Technology, Wilmington, USA) was used. The mobile phase consisted in a mixture of $10 \mathrm{mM}$ phosphate buffer $(\mathrm{pH} 8.0) /$ acetonitrile $(70: 30, \mathrm{v} / \mathrm{v})$. The mobile phase flow rate was $1.0 \mathrm{~mL} \cdot \mathrm{min}^{-1}$. The column temperature was $25^{\circ} \mathrm{C}$. The injection volume was $2 \mu \mathrm{L}$. The detection wavelength was $220 \mathrm{~nm}$. Details of the chromatographic system and sample treatment prior to injection into the chromatographic system are described elsewhere [15].

\subsection{Overall Precision Studies}

Full method validation of the current analytical method becomes out of the "fit-for-purpose concept. The reason are: (i) the method was not designed for routine sample analysis, and(ii)the objective is not to calculate concentrations (calibration is avoided), but biodegradation data directly from peak areas measurements (see section 2.5). On the other hand, performance features of the same procedure has been established elsewhere for two drugs [15], and substantial differences are not expected. In contrast, a study involving the overall (biodegradation and analytical processes) precision becomes more relevant. Repeatability and intermediate precision were estimated adapting methods from the analytical chemistry literature [16,17]; substituting concentration by biodegradation data. Concretely, the biodegradation $(B D)$ data after 7 days of incubation $\left(B D_{7 \mathrm{~d}}\right)$ from duplicate assays $(\mathrm{Nr}=2$ replicates) of seven inoculums (different periods; runs) were submitted to ANOVA to estimate the residual $\left(M S_{\mathrm{r}}\right)$ and between-run $\left(M S_{\text {run }}\right)$ mean square statistics (variance terms). Then, the corresponding relative standard deviation values, $R S D_{\mathrm{r}}$ and $R S D_{\text {run, }}$, were calculated:

$$
\begin{aligned}
& R S D_{r}=\frac{100 \cdot \sqrt{M S_{r}}}{B D_{7 \mathrm{~d}}} \\
& R S D_{\text {run }}=100 \cdot \frac{\sqrt{\frac{M S_{r u n}-M S_{r}}{N r}}}{B D_{7 \mathrm{~d}}}
\end{aligned}
$$

\subsection{Biodegradation Kinetics}

$B D$ data at prefixed times $(t)$ were estimated from measured chromatographic peak areas $\left(A ;\right.$ where $A_{0}$ correspond to $A$ at $t=0)$, as reported in [15]:

$B D=\frac{A_{0}-A}{A_{0}} \times 100$

Eqn(3) was also used for abiotic degradation $(D)$. Biodegradation vs. time fitted curves to the experimental $B D$ - $t$ data were modelled to an adjusted Michaelis-Menten-like equation derived in [15]:

$$
B D=\frac{100}{S_{0}}\left\{S_{0}-K_{s} \cdot W\left[\frac{S_{0}}{\mathrm{~K}_{\mathrm{s}}} \mathrm{e}^{\left(\frac{\mathrm{s}_{0}-V_{\mathrm{m}} \cdot t}{K_{\mathrm{s}}}\right)}\right]\right\}
$$

Where $V_{\mathrm{m}}$ (maximum specific growth rate) and $K_{\mathrm{s}}$ (half saturation constant) are the model parameters to be estimated, $\mathrm{S}_{0}$ is the initial concentration of compound and $W$ represent the Lambert $W$ function [18-20].Iterative least squares parameter estimation was performed using NLINFIT.m function in MATLAB® R2016b (Math Works, Natick, Massachusetts).

\section{RESULTS AND DISCUSSION}

\subsection{Comparison of Potential Compounds to Control the Inoculum Activity}

In previous assays, the use of sodium benzoate and aniline, recommended in the OECD RBTs documents, were evaluated as reference compounds to control the inoculum activity. For sodium benzoate, $B D=80.8 \%$ was reached in $24 \mathrm{~h}$, and biodegradations was almost complete in 3 days; too fast compared to the behaviour of most drugs. In addition, a significant physicochemical degradation was observed ( 22 and $46 \%$ in 3 and 7 days). Similar behaviour was obtained for aniline. These facts make their use no representative as control in drugs biodegradability assays. Sodium acetate, also recommended, was not tested since it was not possible to detect it in HPLC with an UV/Vis detector. 
Because drugs are commonly tested in biodegradability assays, the use of a pharmaceutical instead of the OECD recommended reference compounds was considered a more suitable option. The selected compound should have a moderate biodegradation ( $B D$ over $60-70 \%)$ and a negligible abiotic degradation $(D<20 \%)$. The first level reach the RBT levels to declare a compound as readily biodegradable [4]. The last one means that abiotic elimination of the compound can be considered non-significant [21].It is also preferable that the degradation process is not too rapid, which would enable to obtain a biodegradation curve and its use in a short-term (several days) assay.

Following these two criteria, several biodegradation assays using ten drugs including antihistamines, antidepressants and $\beta$-blockers were performed. Table 1 shows the degradation $\left(D_{7 \mathrm{~d}}\right)$ and biodegradation $\left(B D_{7 \mathrm{~d}}\right)$ results obtained from eqn(3) after 7 days of incubation, corresponding to abiotic and biotic assays, respectively. As can be observed, clemastine and terfenadine show considerable abiotic degradation, 40 and 26\%, respectively, and terfenadine presents too large biotic biodegradation. For these reasons these compounds were refused. On the other hand, labetalol, propranolol, metoprolol, fenfluramine, nomifensine and verapamil, despite having small values of abiotic degradation, they also show relatively low biodegradation after 7 days of incubation, which prevents their use as reference compound.

Fluoxetine was the only compound that fitted the criteria previously indicated. A solution of $20 \mathrm{mg} . \mathrm{L}$ ${ }^{1}$ fluoxetine exhibited moderate biodegradation in 7 days $\left(B D_{7 \mathrm{~d}}=60 \%\right)$ and relatively low degradation in absence of inoculum $(D<20 \%)$. Thus, the possibility of using fluoxetine as an inoculum activity control was further evaluated systematically.

\subsection{Fluoxetine as Reference Compound to Control the Inoculum Activity. Acceptance Criterion}

To evaluate the repeated use of fluoxetine as inoculum activity control, a systematic long-term study, which include habitual source of variation, was performed. The study, which includes seven independent biodegradation experiments, was carried out using four inocula collected from the same WWTP at different dates (from November 2016 to April 2017), different storage times of the inoculum in the laboratory (from 6 to 24 days before use) and three different operators (see Table 2). In all the experiments, the fluoxetine concentration assayed was around $20 \mathrm{mg} \cdot \mathrm{L}^{-1}$. Fig. 1 shows the box plot of the $B D_{7 \mathrm{~d}}$ results. The individual results corresponding to the experiment number in Table 2 are also plotted. The median of these $B D$ results is $(70 \pm 11) \%$, where, the error term was estimated according to the Median Absolute Deviation estimate (MADe; a robust standard deviation [22].

From the results, a criterion of fluoxetine- $B D_{7 \mathrm{~d}}=70 \pm 11$ for inoculum activity control for futuredrug assays is proposed. Values below or above this range could serve to classify the inoculum as low- or high-active, respectively. For instance, an inoculum obtained after a rain period, which could present afluoxetine- $B D_{7 \mathrm{~d}}<59 \%$, should be considered as alowactive inoculum and therefore, the biodegradation data derived from its use should be discarded. In an experiment carried out using a low active inoculum (fluoxetine- $B D_{7 \mathrm{~d}}=48 \%$ ), the $B D_{7 \mathrm{~d}}$ values obtained for the new drugs: trimeprazine, methotrimeprazine and promethazine, were 32,36 and $26 \%$, respectively. The same experiment performed with a normal active inoculum (fluoxetine- $B D_{7 \mathrm{~d}}=78 \%$ ) provided $B D_{7 \mathrm{~d}}$ values of 81,77 and $78 \%$ for trimeprazine, methotrimeprazine and promethazine, respectively.

As can be seen, the application of the fluoxetine- $B D_{7 \mathrm{~d}}$ acceptance criterion is a good indicator of the inoculum activity. During the preparation of this work, the inoculum corresponding to point 7 in Fig. 1 was used to obtain biodegradation curves of two other drugs. ${ }^{15}$ Since the $B D_{7 \mathrm{~d}}$ value of fluoxetine fitted the acceptance criterion proposed (i.e. normal inoculum biodegradation activity), the $B D$ data measured for these drug were considered satisfactory. In addition, fluoxetine as reference compound could be useful to compare/discard different assays/procedures or biodegradation conditions using the same inoculum, or to evidence a time or WWTP variability effects.

\subsection{Long-Term Variability of the Biodegradability Potential of an Activated Sludge (Intermediate Precision Study)}

The precedent results in Fig. 1 suggest that no particular trends were observed with different inoculum collection dates, storage times or operators. On the other hand, if convenient, the activity of the activated sludge of a WWTP plant to be used indrug biodegradability assays could be monitored using fluoxetine as reference compound, e.g. using a control chart. For instance, the centreline of this chart 
Biodegradability Features of Fluoxetine as a Reference Compound for Monitoring the Activity of Activated Sludges in Drug Biodegradation Studies

could be $B D=70 \%$ while the upper and lower control limits could be placed in $70 \pm 11$ (i.e. 59 and $81 \%$ ). In that case, experiment 1 in Table 2 (with $B D_{7 \mathrm{~d}}=56 \%$ ) would be an out-of-control output (the rest of results would be in-control).

On the other hand, the variability of the activated sludge of the current WWTP can be quantitatively established by means of a precision study using eqn(1) and (2).RSD estimates from these equations accounts for both, the biodegradation and the analytical processes. The $R S D_{\mathrm{r}}$ value for $B D_{7 \mathrm{~d}}$ was $7.1 \%$ (repeatability). The $R S D_{\text {run }}$ value was $13.9 \%$ (intermediate precision), which includes variation sources such as different activated sludges collected at different dates, different storage times of the activated sludges in the laboratory and different operators. The relatively low $R S D_{\text {run }}$ result would indicate that the activity of the microbial community remains relatively stable in the WWTP considered in this study. Such $R S D$ data (in repeatability and/or intermediate precision conditions) could be useful to compare precision studies results obtained with different biodegradation assays/procedures or biodegradation conditions or using activated sludges from different treatment plants.

Table1. Degradation $\left(D_{7 d}\right)$ and biodegradation $\left(B D_{7 d}\right)$ values after 7 days of incubation obtained from eqn $(3)$ of the tested drugs for the abiotic and biotic assays, respectively. Concentration of all compounds was approximately $20 \mathrm{mg} \mathrm{mL}^{-1}$

\begin{tabular}{|l|c|c|}
\hline Compound & $D_{7 \mathrm{~d}}(\%)$ & $B D_{7 \mathrm{~d}}(\%)$ \\
\hline Labetalol & 13 & 32 \\
\hline Metoprolol & 8 & 13 \\
\hline Propranolol & 0 & 22 \\
\hline Clemastine & 40 & 48 \\
\hline Fenfluoramine & 7 & 12 \\
\hline Fluoxetine & 0 & 60 \\
\hline Nomifensine & 8 & 19 \\
\hline Orphenadrine & 14 & 25 \\
\hline Terfenadine & 26 & 90 \\
\hline Verapamil & 0 & 11 \\
\hline
\end{tabular}

Table2. Biodegradationexperiment details for the evaluation of fluoxetine as reference substance.

\begin{tabular}{|c|c|l|c|c|}
\hline Experiment & Inoculum & Collection date & Storage time (days) & Operator \\
\hline 1 & 1 & November 11, 2016 & 11 & 1 \\
\hline 2 & 2 & February 2, 2017 & 7 & 1 \\
\hline 3 & & & 10 & 1 \\
\hline 4 & & & 20 & 1 \\
\hline 5 & & & 24 & 1 \\
\hline 6 & 3 & Mach 3, 2017 & 10 & 2 \\
\hline 7 & 4 & April 21, 2017 & 6 & 3 \\
\hline
\end{tabular}

\subsection{Biodegradation Curves of Fluoxetine. Modelling the Biodegradation Data}

To complete the biodegradability features of fluoxetine, its short-term biodegradation profile over time were evaluate. Using two different inocula corresponding to points 5 and 7 in Fig. 1 (where inoculum collection dates, storage times and operators differ), biodegradation assays for fluoxetine were performed. From the assays, $B D$ and $O D_{600}$ (as indicator of the microbial growth [23]) data over time were obtained. Fig. 2 shows the $O D_{600}$ results obtained for the biotic assays and for a blank (with only inoculum). As can be observed, the results for the biotic assays present the individual phases of a typical batch growth curve [23]: lag ( 2 days), exponential ( $\sim 5$ days) and stationary/decline (after day 7) growth phases. As can be expected, the microbial growth in the blank (without fluoxetine) was negligible.

Fig.3 shows the experimental $B D$ data curve combining the results obtained for both fluoxetine biodegradation assays. As can be observed, the results of both assays are reasonably comparable. The whole $B D$ vs. $t$ experimental data were fitted to eqn (4). As expected, the fitted curve shows a value of $B D$ around $70 \%$ at $t=7$, consistently with the experimental $B D_{7 \mathrm{~d}}$ median value at this time. Complete 
Biodegradability Features of Fluoxetine as a Reference Compound for Monitoring the Activity of Activated Sludges in Drug Biodegradation Studies

biodegradation is achieved after 10 days. According to the fitted biodegradation versus time fitted curve in Fig.3, the estimated half-life (tvalue at $B D=50 \%$ ) for fluoxetine is 5 days. This result is in agreement with this previously reported for a $1 \mathrm{mg} \cdot \mathrm{L}^{-1}$ fluoxetine solution [24].Parallel abiotic assays indicate relatively low degradation in absence of inoculum $(<20 \%)$ in the same period of time. The obtainment of a complete biodegradation profile for fluoxetine as reference compound assures adequate inoculum activity during the whole experiment (i.e. a short-term monitoring during several days).

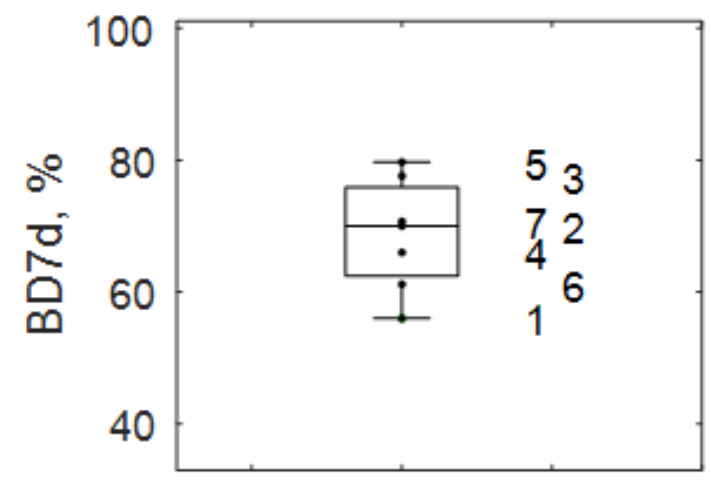

Fig1. Biodegradation results (box plot) after 7 days of incubation $\left(B D_{7 d}\right)$ of $\sim 20 \mathrm{mg} \cdot L^{-1}$ solutions of fluoxetine. Individual BD values (small points) and their corresponding experiment number (in Table 2) are included.

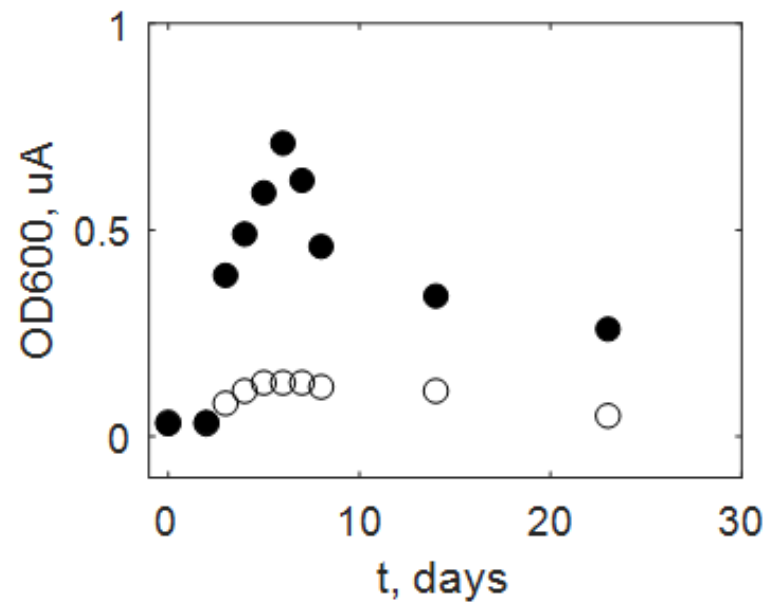

Fig2. Optical density at $600 \mathrm{~nm}\left(O D_{600}\right)$ along the biodegradability assays for:( $)$ fluoxetine biotic assays (initial concentration $\sim 20 \mathrm{mg} \mathrm{L}^{-1}$ ) and, (O) blank.

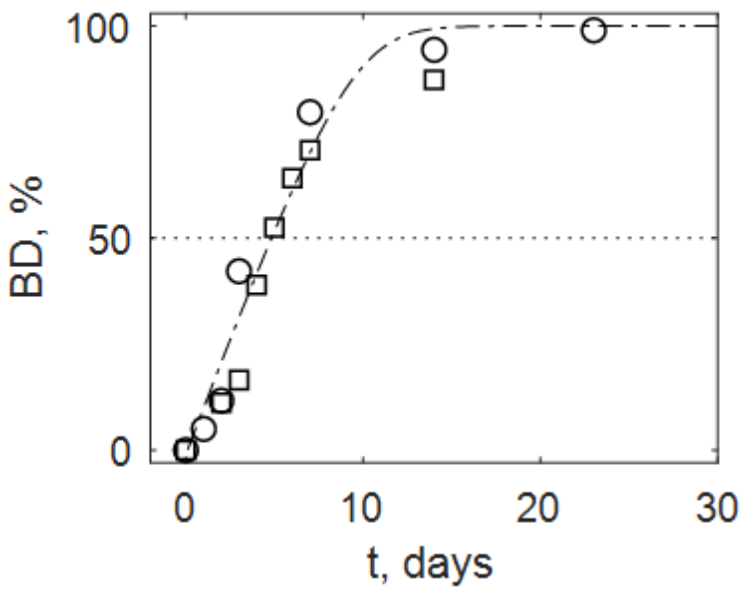

Fig3. Biodegradation versus time (BD vs. $t$ ) experimental data obtained using two different inocula (experiments $5(\mathrm{o})$ and $7(\square)$ in Table 2) and fitted curve (eqn (4)) to the whole combined data (solid line). The initial fluoxetine concentrations were 18.4 and $23.0 \mathrm{mgL}^{-1}$, respectively. 


\section{Conclusions}

Fluoxetine shows suitable biodegradability features to be used as a reference compound to control the inoculum activity over the recommended compounds by the OECD. It shows a biodegradation behaviour more similar to the tested drugs than the usually recommended reference compounds. Its abiotic degradation is negligible $(<20 \%)$ and the complete biodegradation is achieved after 10 days, which enable to obtain a biodegradation curve, useful in comparison studies(e.g. half-life time or curve shape respect to other drugs) and to ensure the adequate inoculum activity when obtaining biodegradation curves for new drugs. In addition, because of fluoxetine is a chiral compound, it could be used to control the inoculum activity in both achiral and chiral biodegradation studies.

The current results, accounting for intermediate precision variability, define aninoculum activity acceptance criterion based on fluoxetine biodegradation after 7 days of incubation ( $70 \pm 11$ range). It indicates the expected normal inoculum activity able to produce valid biodegradability measurements for new drugs. Both, this range and the relative standard deviation obtained in the precision study, could be useful in future comparison studies involving different procedures, conditions, activated sludges, etc.

\section{ACKNOWLEDGEMENTS}

The authors acknowledge the Spanish Ministerio de Economía y Competitividad (MINECO) and the European Regional Development Fund (ERDF) for the financial support (Project CTQ2015-70904-R, MINECO/FEDER, UE).

\section{REFERENCES}

[1] M.D. Hernando, M. Mezcua,A.R. Fernández-Alba,D. Barceló, Environmental risk assessment of pharmaceutical residues in waste water effluents, surface waters and sediments, Talanta69 (2006)370-376.

[2] M.D. Hernando, A.R. Fernández-Alba, R. Tauler andD. Barceló, Toxicity assays applied to waste water treatment,Talanta65 (2005)358-366.

[3] G. Thouand, Biodegradability assessments of organic substances and polymers,Environ. Sci. Pollut. Res.21 (2014)9443-9444.

[4] OECD, 1992, OECD Guideline for testing of chemicals. 301 Ready Biodegradability test.

[5] K. Kümmerer, The presence of pharmaceuticals in the environment due to human use- present knowledge and future changes, J. Environ. Manag. 90 (2009)2354-2366.

[6] C.S. Wong, Environmental fate processes and biochemical transformations of chiral emerging organic pollutants, Anal. Bioanal. Chem. 386 (2006)544-558.

[7] J.K. Stanley, A.J. Ramirez, M. Mottaleb, C.K. Chambliss,B.W. Brooks, Enantiospecific toxicity of the $\beta$ blocker propranolol to Daphnia magna and Pimephales promelas, Environ Toxicol. Chem. 25 (2006)17801786.

[8] J.K. Stanley, A.J. Ramirez, C.K. Chambliss,B.W. Brooks. Enantiospecific sublethal effects of the antidepressant fluoxetine to a model aquatic vertebrate and invertebrate, Chemosphere 69 (2007)9-16.

[9] B. Kasprzyk-Hordern, Pharmacologically active compounds in the environment and their chirality, Chem. Soc. Rev.39 (2010)4466-4503.

[10] A.R. Ribeiro, P.M.L. Castro, M.E. Tiritan. Environmental Fate of Chiral Pharmaceuticals: Determination, Degradation and Toxicity,in: E. Lichtfouse, J. Schwarzbauer, D. Robert (Eds.),Environmental Chemistry for a Sustainable World, Springer, Dordrecht, 2012,pp. 3-45.

[11] G.A. Vázquez-Rodríguez, R.I. Beltrán-Hernández, C. Coronel-Olivares,J.L. Rols. Standardization of activated sludge for biodegradation tests,Anal. Bional. Chem.401 (2011)1127-1137.

[12] K. Liao, Y. Bai, Y. Huo, Z. Jian, W: Hu, C. Zhao, J. Qu, Integrating microbial biomass, composition and function to discern the level of anthropogenic activity in a river ecosystem, Environ. Int.116 (2018)147155.

[13] B.D.H. Phuc, S.-S. You, B.M. Hung, H.-S. Kim, Robust control synthesis for the activated sludge process, Environ. Sci.: Water Res. Technol.4 (2018)992-1001.

[14] L. Escuder-Gilabert, Y. Martín-Biosca, M. Perez-Baeza, S. Sagrado andM. J. Medina-Hernández, Trimeprazine is enantioselectively degraded by an activated sludge in ready biodegradability test conditions, Water Res.141 (2018)57-64.

[15] L. Escuder-Gilabert, Y. Martín-Biosca, M. Perez-Baeza, S. Sagrado, M.J. Medina-Hernández, Direct chromatographic study of the enantioselective biodegradation of ibuprofen and ketoprofen by an activated sludge,J. Chromatogr., A 1568 (2018)140-148. 
Biodegradability Features of Fluoxetine as a Reference Compound for Monitoring the Activity of Activated Sludges in Drug Biodegradation Studies

[16] E. Bonet-Domingo, L. Escuder-Gilabert, M. J. Medina-Hernández, S. Sagrado,Uncertainty-Based Internal Quality Control. Harmonization Considerations, Anal. Chem.78 (2006) 8113-8120.

[17] A. Maroto, J. Riu, R. Boqué, F.X. Rius, Estimating uncertainties of analytical results using information from the validation process, Anal. Chim. Acta391 (1999)173-185.

[18] C.T. Goudar, K.A. Strevett, Estimating in-situ Monod biodegradation parameters using a novel explicit solution of a one-dimensional contaminant transport equation, Ground Water 38 (2000)894-898.

[19] R.M. Corless, G.H. Gonnet, D.E. Hare, D.J. Jeffrey, On the Lambert W function. Technical Report Cs-9303. Waterloo, Canada: Department of Computer Science. University of Waterloo, 1993.

[20] C. Her, A.P. Alonzo, J.Y. Vang, E. Torres, V.V. Krishnan, Real-Time Enzyme Kinetics by Quantitative NMR Spectroscopy and Determination of the Michaelis-Menten Constant Using the Lambert-W Function,J. Chem. Educ. 92 (2015)1943-1948.

[21] ISO 9888:1999. Norma UNE-EN ISO 9888. Calidad del agua. Evaluación de la biodegradabilidad aeróbia final de los compuestos orgánicos en medio acuoso. Ensayo estático (Método de Zahn-Wellens). AENOR, Madrid, España, 1999.

[22] The Royal Society of Chemistry. http://www.rsc.org/Membership/Networking/Inte rest Groups/Analytical/ AMC/Software/RobustStatistics.asp, (Accessed 22 October 2018).

[23] M.L. Shuler, F. Kargi,Bioprocess Engineering: Basic Concepts, second ed., Prentice Hall, Upper Saddle River, NJ, 2012.

[24] A.R. Ribeiro, C.M. Afonso, P.M.L. Castro, M.E. Tiritan, Enantioselective HPLC analysis and biodegradation of atenolol, metoprolol and fluoxetine, Environ. Chem. Lett.11 (2013) 83-90.

Citation: Yolanda Martín-Biosca et al.. " Biodegradability Features of Fluoxetine as a Reference Compound for Monitoring the Activity of Activated Sludges in Drug Biodegradation Studies" International Journal of Advanced Research in Chemical Science (IJARCS), vol. 6, no. 1, pp. 16-23, 2019. http://dx.doi.org/10.20431/ 2349-0403.0601003

Copyright: (C) 2019 Authors. This is an open-access article distributed under the terms of the Creative Commons Attribution License, which permits unrestricted use, distribution, and reproduction in any medium, provided the original author and source are credited. 